\title{
Multilinguales
}

\section{La traduction, une problématologie : l'horreur dans Murambi, le livre des ossements de B. B. Diop}

Translation, a Problematology: Horror in Murambi, le Livre des Ossements by Boubacar Boris Diop

\section{Baboucar Diouf}

\section{(2) OpenEdition}

\section{Journals}

Édition électronique

URL : http://journals.openedition.org/multilinguales/949

DOI : $10.4000 /$ multilinguales.949

ISSN : 2335-1853

\section{Éditeur}

Université Abderrahmane Mira - Bejaia

Édition imprimée

Date de publication : 1 juin 2015

Pagination : 47-63

ISSN : 2335-1535

\section{Référence électronique}

Baboucar Diouf, « La traduction, une problématologie : l'horreur dans Murambi, le livre des ossements de B. B. Diop », Multilinguales [En ligne], 5 | 2015, mis en ligne le 01 juin 2015, consulté le 17 septembre 2019. URL : http://journals.openedition.org/multilinguales/949; DOI : 10.4000/multilinguales.949

Ce document a été généré automatiquement le 17 septembre 2019.

\section{cc) (†)}

Multilinguales est mise à disposition selon les termes de la Licence Creative Commons Attribution -

Pas d'Utilisation Commerciale - Pas de Modification 4.0 International 


\section{La traduction, une problématologie : l'horreur dans Murambi, le livre des ossements de B. B. Diop}

Translation, a Problematology: Horror in Murambi, le Livre des Ossements by Boubacar Boris Diop

Baboucar Diouf

1 Dans cette contribution, nous étudierons la traduction anglaise de Murambi, le livre des ossements (désormais MLO) de Boubacar Boris DIOP, par Fiona Mc LAUGHLIN, intitulée Murambi : the Book of Bones (MBB). Nous nous intéressons, en particulier, au traitement de la narration de l'horreur du génocide rwandais. Pour comprendre les transformations narratives que subit le texte source par rapport au texte traduit, nous nous sommes référés à la théorie du sens et aux théories inter-linguistiques en rapport avec notre problématologie. Cet article exploite la traduction d'abord dans sa fidélité, puis dans son infidélité au texte premier.

La théorie du sens fonde son argument sur la transplantation du sens du texte source dans le texte cible, c'est-à-dire sur la restitution du sens et sur l'équivalence entre la traduction et le texte traduit.

Nous exploitons aussi la traduction de Mc Laughlin sous l'angle de la polémique entre Henry Meschonnic et Jean-René Ladmiral. Le premier négocie la distance entre le fond et la forme, le sens et le style et privilégie le discours (la traduction discursive). Le second, axe sa réflexion sur la traductologie pour opposer lettre et sens et partage la conception de son approche avec les sémanticiens de la traduction :

Surtout, il apparaît qu'en traductologie, il y a deux traditions opposées: celle dont la poétique de la traduction développée par $H$. Meschonnic est à mes yeux un exemple, et celle que j'appelle les sémanticiens de la traduction, à laquelle je me rattache moi-même [...] et dont G. Mounin est lui-même un exemple. (J.-R. Ladmiral, 1981 : 15)

2 Ces approches, surtout celles du sens et celle de la sémantique sont mises à profit pour analyser le versant problématologique de la traduction. 
D'abord, avec la théorie du sens, la traduction met au menu des enchaînements de changements narratifs complexes non négligeables attestant que le recours au langage obéit à certaines règles rhétoriques relatives à la théorie du questionnement de Michel Meyer.

Ensuite, avec la théorie inter-linguistique en rapport avec la sémantique des possibles argumentatifs, la traduction de Mc Laughlin expose l'imputabilité d'une infidélité au texte source. Dans ces contextes, le langage exprime soit une question, soit une réponse et expose des problèmes et des solutions déclaratives ou non déclaratives. Alliée à la traduction, la problématologie exploite la complexité des changements qui dépendent du passage d'une langue à l'autre.

Dans cet article, la traduction pose le problème de la prise de parole et du destinataire du discours dans la transposition de la mémoire génocidaire des rescapés rwandais. La traduction semble faire éclore la problématologie de l'identité des narrateurs, des personnages, des lecteurs et des auteurs. Cette complexité identitaire pose nécessairement le problème de l'analyse d'un texte second traduit par rapport à la version initiale. La confusion s'invite et interpelle la critique, car d'une version à une autre, le style, le lecteur, la sensibilité, le narrateur, le narrataire, etc., peuvent être différents de leurs vis-à-vis initiaux. D'où la problématologie de la traduction, la seconde version étant une réponse à la question non posée de l'intérêt à avoir une version dans une autre langue. C'est la raison pour laquelle l'imputabilité de fidélité ou d'infidélité au texte originel nécessite une étude approfondie.

\section{L'imputabilité d'une fidélité au texte source}

Dans Murambi, the Book of Bones, la traduction objective de la subjectivité expose les formes d'énonciation argumentative que l'on retrouve dans la version originale. C'est l'inscription de celui qui énonce qui marque l'empreinte de la fidélité au texte initial. À ce niveau, les marqueurs linguistiques qui permettent la reproduction objective de la subjectivité des énonciateurs dans la version traduite transmettent le rapport existant entre énonciateur / message / énonciataire. Il en résulte une reproduction presque parfaite du schéma d'échange de départ :

\begin{tabular}{|c|c|c|c|c|c|c|}
\hline Texte original & $\rightarrow$ & Énonciateur 1 & $\rightarrow$ & Énonciateur 1 & $\rightarrow$ & Énonciataire 1 \\
\hline$\downarrow$ & & $\downarrow$ & & $\downarrow$ & & $\downarrow$ \\
\hline Texte traduit & $\rightarrow$ & énonciateur2 & $\rightarrow$ & message2 & $\rightarrow$ & énonciataire2 \\
\hline
\end{tabular}

Dans la traduction, nous pouvons relever l'expression de fidélité sur la base du recours à l'expressivité objective et subjective. Cette dernière passe, par exemple, par la reproduction du «je» (Diop, 2000:9) - «I» (Laughlin, 2006:19) et d'autres types de marqueurs tels que «moi-même» (Diop, Ibid.) - «myself» (Laughlin, Ibid.). Sur ce plan, nous pouvons dire que le vocabulaire axiologique, impliquant la présence des jugements de valeurs (adverbes et adjectifs évaluatifs), traduit la force de l'écriture des inédits historiques :

I've seen lots of scenes on television myself that were hard to take. Guys in slips and masks pulling bodies out of a mass grave. Newborns they toss, laughing, into bread ovens. Young 
women who coat their throats with oil before going to bed. "That way," they say, "when the throat-slitters come, the blades of their knives won't hurt as much." (Laughlin, 2006 : 19-20) ${ }^{1}$

L'adjectif « hard» exprime le poids de l'horreur consubstantiel à l'écriture tragique. La traduction de ces genres de marqueurs affectifs entraîne celle de l'identité psychologique exprimant les marques de la reproduction des désignations des caractères des personnages. Outre cela, l'identité ethnique, à l'origine de la tragédie, est reconduite dans la seconde version qui agit comme vecteur testimonial de l'expérience du génocide :

Well, it was at Gitarama, where we Hutus were the strongest. While our people were busy looting and raping, a four-year-old child and his parents were waiting for a car in order to escape toward Mutara. Suddenly, our men saw the Inyenzi family hurrying to get into the car. They ran after them. But it was too late. That's how those imbeciles, thirty-seven years ago, let the kid who is now the head of the guerrilla force escape (Laughlin, $2006: 16)^{2}$.

Ici, la différence ethnique entre Hutus et Tutsis apparaît sous le sceau de la distanciation empreinte d'une haine génocidaire soucieuse de l'élimination de la mémoire des témoins. L'ethos du cynisme des Hutus fonde l'écart entre Hutus et Tutsis : Hutu et Tutsi seraient comme deux espèces humaines vouées à s'entredéchirer en vertu d'un atavisme irrépressible. Ce cliché se double en général d'un manichéisme pseudo moral consistant à identifier en bloc les bons et les méchants (Chrétien, 1995 : 45).

5 Au-delà de la justification du génocide par les Hutus, le texte reproduit l'esprit de résistance des Tutsis :

Ever since 1959, every young Rwandan, at one moment or another in his life, has to answer the same question: Should we just sit back and wait for the killers, or try to do something so that our country can go back to being normal? (Laughlin, $2006: 31)^{3}$.

Dans l'énonciation argumentative de l'objectivité, le traducteur reconduit le refus d'effacement de la mémoire. Mc Laughlin présente, comme c'est le cas dans le texte initial, les faits sous l'angle de la scientificité avec une distanciation sous forme de réinitialisation des mêmes données dans la langue anglaise. Sous ce rapport, l'argument d'autorité s'appuie sur la perte du président rwandais et de l'autorité de Dieu, tel que présenté explicitement dans le texte source pour calquer la mythologie rwandaise et la doxa (Laughlin, 2006 : 11).

De la même manière que le traducteur reproduit les différents énonciateurs, il décrit l'inscription de l'énonciataire dans son texte. Ce dernier s'inscrit dans le texte romanesque. De ce fait, l'inscription se fait par l'empreinte du marqueur linguistique (langue française dans la version originale et langue anglaise dans la version traduite) : ML1 = ML2. Cette identification inscrit dans le même temps l'identité de l'énonciataire pour laquelle nous notons par fidélité de la traduction qu'il y a plusieurs rapports entre narrateur et narrataire. Ces rapports nous amènent à étudier les formes d'énonciation narrative. C'est le lieu où il faut noter la fidélité à la plurivocité du texte initial.

8 Notre option consiste à considérer que dans le cas de la réécriture de Mc Laughlin, le narrateur de chaque histoire se comporte comme narrateur-témoin. En sa qualité de témoin tutsi, Michel Surumundo, par exemple, exprime sa peur et l'horreur de la tragédie. Le second narrateur, Faustin Gasana, membre de la milice Interahamwe, demande que l'on tue tous les Tutsis :

It's true, if we don't succeed in eliminating all the Tutsi, we'll be considered the bad guys in this business. They'll trot out their sob stories before the entire world and things will get very complicated for us. Even the most reluctant among us know it : after the first machete blow, we'll have to see it through to the bitter end (Laughlin, $2006: 16)^{4}$. 
La polyphonie narrative qui désigne les narrateurs comme des narrateurs homodiégétiques déploie les différentes formes de tonalité. De ce fait, la traduction de Mc Laughlin est fidèle à la dispositio du texte originel, car elle reprend scrupuleusement les formes de focalisation. Dans le texte traduit, la focalisation est non médiatisée, car les narrateurs assurent la focalisation. Ils donnent accès à l'information en suivant leurs propres points de vue et livrent l'information telle qu'ils sont en mesure de la percevoir (Laughlin, 2006). La traduction de Mc Laughlin est donc fidèle à la reprise de la dispositio et de la narrativité (description, discours rapporté, structure temporelle et la mise en abyme dans le texte originel).

Avec la réécriture de la description, le traducteur reproduit aussi l'environnement dans lequel se déroule l'histoire (Rwanda). Cela correspond à la description du contexte du génocide (description des lieux et des décors à l'époque de l'ethnocide) qui renvoie à la fidélité à l'histoire, à l'intrigue et à l'action.

La traduction de Mc Laughlin suit les lignes historiques tracées par le texte de Diop. Le traducteur établit les liens logiques et de causalité comme l'exige une traduction fidèle à la représentation de l'histoire du génocide et du «cynisme » des Hutus. Suivant le même processus de reprise de l'histoire, le traducteur reproduit fidèlement les épisodes, les incidents et les motifs. Ces deux aspects du récit se lisent dans l'action. Nous notons à ce niveau que l'on retrouve la même architecture textuelle du texte source dans le texte traduit. Cela veut dire qu'il existe un rapport strictement rhétorique entre ces différentes composantes. Donc l'histoire, l'intrigue et l'action sont des invariables rhétoriques dans la traduction.

D'après ces différents aspects, nous pouvons retenir que la traduction est un art qui vise à reproduire l'unité de sens (Us) d'une langue originelle (Lo) dans une langue d'arrivée (La) ; attendu qu'une autre version (Vn) peut s'inspirer de la langue d'arrivée (La). Relatant les événements du génocide de 1994, Murambi, the Book of Bones atteste sa fidélité au texte source grâce à la reprise de la narration de l'horreur. Cette version (La) est une reproduction fidèle aux périodes et séquences de la (Lo). La version anglaise dévoile une fidélité à l'originale. Cette fidélité s'exprime d'ailleurs de façon éloquente au niveau de la reproduction de l'histoire, de l'intrigue et de l'action dans la narrativité du roman initial. L'idée est d'élargir l'éventail des possibilités de gagner un lectorat pour diffuser l'histoire de la tragédie génocidaire au-delà de la frontière linguistique de (Lo).

Cependant, dans cette fidélité, la version anglaise de Mc Laughlin négocie la distance des unités de sens (Us) entre la (Lo) et la (La). L'identification "résout à sa façon le problème d'une distance » qui pourrait, dans un autre versant de la critique (Benjamin, $2000: 16)$, « s'affirmer et se confirmer » (Meyer, $2004: 14$ ).

La version originelle constitue l'ad rhem, c'est-à-dire la question, et la version traduite représente l'ad hominem, la touche personnelle. L'ethos de la (La) s'identifie alors à l' ethos de la (Lo). De ce fait, la fidélité à la version originale se lit dans l'inventio, c'est-àdire l'énonciation argumentative, la réponse à la question traitée.

Le passage du texte source au texte traduit est forcément rhétorique dans la mesure où la langue française transplante le sens du message textuel dans la langue anglaise. De ce fait, la traduction du roman est une réponse (r2) à la question (q2) non posée de la question non posée (q1) de la réponse ( $\mathrm{r} 1$ ), la version originale. Cela veut dire que la traduction respecte la règle générale de la rhétorique proposée par Michel Meyer : $\mathrm{r} 1 \rightarrow$ q1.q2 donc r2. D'où traduire la narration du génocide rwandais, c'est traduire la volonté 
des commanditaires du roman de Boubacar Boris Diop. Ainsi, la traduction de Mc Laughlin est une réponse condensée qui est rhétoriquement équivalente au souci d'exhumer le passé du génocide rwandais: "rhétoriquement, on rend les deux réponses équivalentes, non pas littéralement mais figurativement » (Meyer, 2004 : 53).

En d'autres termes, l'efficacité du discours romanesque second traduit l'efficacité du contenu du texte initial. Cela passe nécessairement par la fidélité expressive de la dispositio, mise en forme et fond de l'adresse rhétorique. L'énonciation argumentative de la traduction fait jouir le texte d'une crédibilité de fidélité au texte original. En somme, par l'inventio le traducteur reprend les formes d'énonciation argumentative suivant la description objective ou subjective où se lit la rhétoricité du discours. Cependant, la traduction de l'histoire du génocide cache une infidélité non moins négligeable.

\section{L'imputabilité d'une infidélité au texte originel}

Dans ce passage, l'analyse va se faire sous le prisme de la traduction issue du principe linguistique en rapport avec la problématologie et la sémantique des possibles argumentatifs. Mais, pour comprendre l'imputabilité de l'infidélité du texte de Mc Laughin, nous allons au préalable, examiner les différentes étapes d'effritement du message génocidaire au terme duquel est né le texte traduit.

L'infidélité au texte originel suit les traces de l'infidélité de la représentation de l'histoire du génocide rwandais par les témoins, l'écrivain et le traducteur.

La narration de la tragédie du génocide rwandais passe par le filtre de l'entretien. Ce filtre romanesque, Murambi, the Book of Bones, obéit aux exigences de la commande du projet de Fest'Africa «Rwanda : écrire par devoir de mémoire ». Le souhait des initiateurs de ce projet était d'amener les auteurs choisis à représenter l'horreur du génocide dans le répertoire des écrits mémoriaux par devoir :

Pour en revenir à Murambi, le livre des ossements, les organisateurs du projet ' $R$ wanda:

écrire par devoir de mémoire' avaient eu l'élégance de nous dire: 'Chacun de vous est

supposé nous remettre un texte à l'issue de la résidence de Kigali mais ne vous croyez pas

obligés d'écrire quoi que ce soit'" (Camara, $2014: 330$ ).

La rhétorique du devoir dans ce souhait, qui laisse lire une liberté de choisir, est l'expression d'une question affirmative dont la réponse idéale se trouve dans la transcription par devoir de transmission de l'histoire du génocide.

De ce devoir, sont nées deux ramifications: un devoir d'écrire par obéissance aux recommandations des initiateurs du projet et un devoir d'écrire par inspiration relative aux témoignages collectés. L'un ou l'autre devoir bute contre la volonté des témoins, la mémoire des victimes, la conscience de l'écrivain et le choix langagier du traducteur pour restituer l'Us du texte de départ, Murambi, le livre des ossements.

Traduit, Murambi, the Book of Bones expose une ossature plus ou moins fidèle à l'histoire réelle vécue par les victimes et leurs bourreaux. Aussi, sous le signe du témoignage, l'histoire du génocide se rapproche plus ou moins de la réalité des faits historiques décrits dans Murambi, le livre des ossements. La même histoire est donc reproduite dans le cadre rédactionnel par l'écrivain, par le biais de la fiction de l'horreur génocidaire, et par le traducteur. Dans un entretien avec Ousmane Ngom et Boubacar Camara, Diop affirme: "au moins un million de Tutsi tués pendant trois mois, ça veut dire plus de 10.000 nouveaux cadavres chaque jour pendant 100 jours et sans un jour d'interruption " (Camara, 2014 : 315-316). Voilà déjà une idée de l'horreur que l'écrivain n'a pas pu/su reproduire 
fidèlement dans son roman. Distance pour distance de réception, de sentiment, de géographie, de temps, et d'appartenance ethnique, le filtre psychologique de l'incidence et de l'indice de culpabilité traverse la problématologie de l'horreur sous la plume traduite. La version originale, tout comme la version traduite, devient le versant-réponse de l'envers-question qui problématise la mémoire du génocide.

Le filtre romanesque de la représentation du génocide rwandais est une trahison avérée par les propos de Diop, lui qui disait se vouloir fidèle à l'histoire génocidaire. Murambi, le livre des ossements est un roman qui exprime par pudeur la cruauté du génocide rwandais. Si traduire veut dire trahir, alors il est naturel de remarquer quelques différences textuelles. La narration de la tragédie du génocide rwandais passe par le filtre de la langue anglaise. Cette version doit sa naissance à la négociation de la distance pour transformer les valeurs en passions. À ce niveau de l'écriture, de l'empreinte de la souffrance, de la mort, de l'horreur dans la psychologie des Tutsis et des Hutus, Diop négocie la distance de la fidélité aux propos de son échantillon et à la teneur du drame orchestré par les camps en conflit. Cette stratégie d'écriture fait perdre ou gagner en originalité ou en fidélité à la traduction du génocide. Cela passe par la transcription écrite telle que schématisée ci-dessous :

Échantillon $\rightarrow$ enquêteur $\rightarrow$ écrivain $\rightarrow$ lecteur $\rightarrow$ traducteur $\rightarrow$ lecteur

Ce schéma est une représentation de la transmission de la mémoire de l'histoire du génocide rwandais selon le rapport des données dans le roman de Diop. Sous le filtre " question-réponse ", l'éthique artistique de Fiona Mc Laughlin se base sur le survol de la tragédie par souci d'actualisation, de transformation, et contradiction du sinistre. Elle essaie de négocier la distance avec la mémoire génocidaire et d'infirmer la possibilité de l'oubli. Le contexte énonciatif sombre dans l'entreprise, à la limite impossible, du rapprochement des faits historiques.

L'histoire refoulée du génocide rwandais dont Diop souligne l'importance dans son roman est projetée dans la traduction de Mc Laughlin. Nous pouvons dire avec Derrida que

[l'on] n'écrit jamais ni dans sa langue ni dans une langue étrangère. [...] Übersetzung et translation surmontent, de façon équivoque, la perte d'un objet. Un texte ne vit que s'il survit, et il ne sur-vit que s'il est à la fois traductible et intraduisible (toujours à la fois, et: ama, en "même" temps). Totalement traductible, il disparaît comme texte, comme écriture, comme corps de langue. Totalement intraduisible, même à l'intérieur de ce qu'on croit être une langue, il meurt aussitôt. La traduction triomphante n'est donc ni la vie ni la mort du texte, seulement ou déjà sa survie. On en dira de même de ce que j'appelle écriture, marque, trace, etc. Ça ne vit ni ne meurt, ça survit. Et ça ne "commence" que par la survie..." (Derrida, 1986 : 147-149).

16 À ce niveau, la version traduite est une métaphorisation du travail romanesque de Diop. Autrement dit, la version traduite, Murambi, the Book of Bones, n'est qu'une autre réponse qui s'inspire de celle de Diop, Murambi, le livre des ossements. La mémoire génocidaire, par le choix des mots, grâce à l'arbitraire de McLaughlin, n'est plus ce qu'elle est dans la production de Boris. Cette traduction ne devient valable par fidélité au texte que métaphoriquement, donc rhétoriquement. Nous sommes ainsi dans la différence problématologique, c'est-à-dire dans l'identité faible, là où le texte traduit devient "une double réponse, dans la mesure où elle avance en se traduisant par la métaphorisation des vieilles réponses, ne les balayant en une fois, ni toutes à la fois » (Meyer, $2004: 87)$. 
17 La «polyénonciation» exprimée fidèlement dans la traduction est une confirmation rhétorique de l'importance de la traduction de la mémoire historique du génocide. La représentation du génocide par le schéma ci-dessus montre la transformation de la forme de transmission du message de la tragédie.

La distance des personnes reproduisant la mémoire du génocide devient, progressivement, plus ou moins proche des faits historiques. Ce qui fait que l'on peut toujours noter une petite distance par rapport à la fidélité aux événements de la part de l'informateur (la personne enquêtée), de l'écrivain (l'enquêteur) aussi bien que du traducteur. De ce fait, le texte gagne ou perd en information d'où :

- L'informateur (les rwandais) restitue 1) fidèlement ou 2) plus ou moins fidèlement les faits historiques par devoir de mémoire ;

- L'écrivain (Boubacar Boris Diop) décrit 1) fidèlement ou 2) plus ou moins fidèlement les faits historiques par devoir de mémoire ;

- Le traducteur (Fiona Mc Laughlin) traduit 1) fidèlement ou 2) plus ou moins fidèlement les faits historiques par devoir de mémoire.

Les informations plus ou moins fidèles aux faits historiques peuvent être représentées sous la forme d'un cube sachant que $\mathrm{I}=$ informateur, $\mathrm{E}=$ écrivain et $\mathrm{T}=$ traducteur :

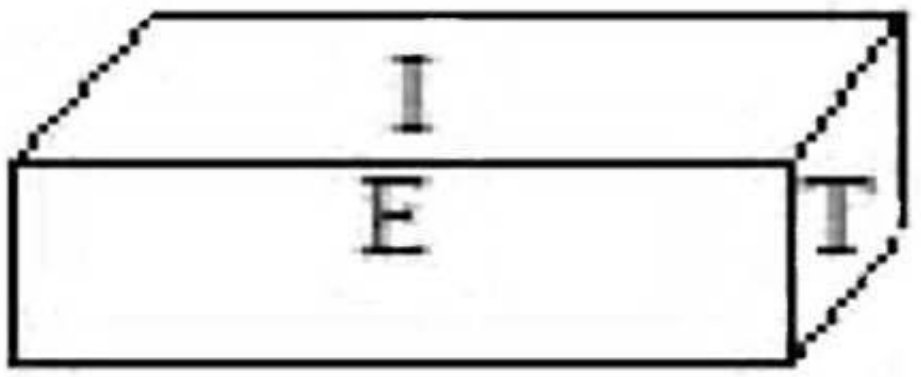

Ce schéma représente les différentes phases par lesquelles la narration du génocide rwandais est passée. Les faces apparentes représentent la mystification de l'histoire, alors que les faces cachées représentent la partie refoulée de l'histoire. Nous avons de ce fait une histoire cachée et une histoire dévoilée. Cette dernière est le problématique alors que la première est l'apocritique, la réponse. Dans tous les cas, l'idée est de faire " entrer le génocide dans la mémoire collective en Afrique et en dehors de celle-ci " (Laughlin, 2006 : 187).

Néanmoins, le domaine source et le domaine cible produisent la même histoire, mais restent profondément différents. Pour qu'il y ait une rhétorique de la traduction du roman de Diop, il a fallu que la question qui suppose que Fiona Mc Laughlin traduise se pose et persiste en dépit de ce qui la résout ou en raison de la langue ou du sens traduit qui la résout (Meyer, 2004 : 51). Cela suppose que le texte d'arrivée (Murambi, the Book of Bones), qui lie l'écrivain (Diop) au traducteur (Mc Laughlin), soit porteur d'autres codes linguistiques ou d'autres significations que ceux du texte traduit.

Pour le montrer, nous prendrons deux exemples sur la base d'un modèle théorique de description lexicale (la sémantique des possibles argumentatifs) en relation avec l'approche de Ladmiral. Cette théorie étudie "les mécanismes discursifs de construction du sens en co-texte et en contexte (faisant) apparaître les phénomènes de déploiement du potentiel argumentatif de la signification lexicale [...] mais également des phénomènes d'affaiblissement 
du potentiel discursif des significations des mots ou de transgression, voire d'intervention de ce potentiel» (Galatu, 2005 : 189).

Dans le travail de traduction des mécanismes sémantico-discursifs, Mc Laughlin trahit la stabilité de l'association à la base du bloc argumentatif suivant :

(1) «Depuis quelques jours mon travail consiste surtout à évacuer sur Bukavu des ministres, des préfets, et des officiers supérieurs» (MLO, 141).

(2) «As of a few days ago my job has been primarily to evacuate ministers, prefects, and superior officers to Bukavu» (MBB, 116).

Dans (2), le mécanisme sémantico-discursif diminue à cause du choix lexical "job » ( "travail») dans la version traduite au lieu de "work» pour activer le potentiel argumentatif présent dans le texte source. Dans la traduction (2), le potentiel argumentatif du noyau du stéréotype y afférent s'en trouve trahi. Ici la génération de la probabilité discursive (Galatu, 2008: 395) s'effrite dans la version traduite à cause du choix du mot «job». De ce fait, dans la version traduite, la gamme d'outils variés à l'expression du mot s'anéantit avec le changement du terme "work » pour «travail » au profit du potentiel argumentatif de « job ». L'infidélité réside dans le changement de l'invariant sémantique par suppression- remplacement; en d'autres termes, dans cette négociation de la distance "significationnelle" suivant le choix lexicographique, l'écart entre le texte originel et le texte traduit confirme l'opposition lettre/sens et l'imputabilité de l'infidélité de la traduction de Mc Laughlin.

Reprenant la traduction du même mot dans un autre passage, l'analyse du mécanisme sémantico-discursif du mot «travail », dans le texte source révèle que la traduction de Mc Laughlin trahit la stabilité de l'association à la base du bloc argumentatif :

(3) «C'est un travail très lent de chacun de nous sur lui-même» (MLO, 59).

(4) «It's a very slow project that each one of us takes upon himsel » (MBB, 48).

Si nous considérons l'activation du potentiel argumentatif de «travail ", l'on remarque naturellement, par application de l'étude sémantico-discursive, que l'activité psychologique n'est pas rendu par le mot «project» dont la teneur discursive serait rendue efficacement avec « work » par souci d'équivalence à la version originale. Dans ce cas, la figuralité du langage pourrait admettre l'option « work » à " project » ouvrant aussi la pertinence de la traduction fidèle de l'occurrence « travail ».

Cependant, dans le cas où le texte traduit ne comporterait aucune anomalie interprétative, ce qui risque moins de se produire, la fidélité au texte original serait avérée. Dans ce cas, la théorie consiste à " comprendre le texte original, à déverbaliser sa forme linguistique et à réexprimer dans une autre langue les idées comprises et les sentiments ressentis » (Lederer, $1994: 11)$. De ce fait, si nous prenons le mot « travail » dans le texte source comme repère, par le caractère pertinent de l'infidélité de sa traduction par «job»(2), nous pouvons retenir que le modèle interprétatif se focalise sur la compréhension - déverbalisation - réexpression (reformulation ou reverbalisation) ou compréhension, reformulation et justification (Delisle, $1980: 85$ ). Cette interprétation des possibles argumentatifs repose sur l'alliance modèle interprétative, étude sémantico-discursive et problématologie.

C'est pourquoi, dans la traduction de Mc Laughlin, le terme « job» est utilisé dans un sens potentiellement argumentatif par négociation des écarts des valeurs linguistiques et culturelles entre l'anglais et le français. En conséquence, par moment, comme c'est le cas ici, Mc Laughlin met en pratique "[l'] opération mentale, qui repose pour son accomplissement sur des processus intellectuels et intuitifs comme la compréhension et le jugement » (Ballard, 1993 : 247). 
Mais si le texte d'arrivée ne permet pas la compréhension littérale de l'original, nous sommes dans le cas d'une rhétorique de la traduction. L'inférentialité des deux textes entraîne, même si la visée première n'est pas celle-ci, un écart d'identification, sauf peut-être, sur le plan littéral.

Dans son travail de traduction, Mc Laughlin négocie la distance avec la mémoire génocidaire en infirmant par voie rhétorique la possibilité de l'oubli. Le contexte énonciatif est reproduit par correspondance dans une logique problématologique déterminante dans l'itération linguistique fidèle au rapprochement des faits historiques. Les récits des témoins oculaires sont repris pour traduire la fidélité à la tragédie ethnocide par ricochet translinguistique dans le texte cible grâce à la reproduction de l'inventio et de l'elocutio.

Cependant, dans le refoulement dérivé du refoulement primaire dans l'entretien et le texte source, l'histoire du génocide devient, dans le texte traduit, une double réponse au problématique non traduit (au premier et au second sens).

$\mathrm{Au}$ terme de cette étude, nous retenons que le texte traduit agit comme une figure dans la mesure où le répondre textuel ou texte traduit subsume le problématique comme si la question de sa différence linguistique et d'unité de sens avec le texte initial était résolue. Ainsi, le texte second abolit l'infidélité, qui semble disparaître, grâce au refoulement de l'original que la transplantation linguistique neutralise.

\section{BIBLIOGRAPHIE}

BALLARD Michel, La traduction à l'université, Lille, Presses Universitaires de Lille, (éd.) 1993.

BENJAMIN W., The Task of the Translator, translated by Harry Zohn, Routledge, ed. Lawrence Venuti, 2000.

CAMARA Boubacar, NGOM Ousmane, « Boubacar Boris Diop : l'écrivain et ses ombres : une Interview », in «Boubacar Boris Diop : une écriture déroutante », in Revue du Groupe d'Études Linguistiques et Littéraires, $n^{\circ}$ 1, Hors-série, avril 2014.

CHRETIEN Jean Pierre, « Un génocide africain : de l'idéologie à la propagande » dans Rwanda un génocide du XXe siècle, R. Verdier, E. Decaux J.-P. Chrétien, l'Harmattan, Paris, 1995.

DELISLE J., L'analyse du discours comme méthode de traduction, Ottawa, Éditions de l'Université d'Ottawa, 1980.

DIOP Boubacar Boris, Murambi, le livre des ossements, Paris, Stock, 2000.

DIOP Boubacar Boris, Murambi, the Book of Bones, translated by Fiona Mc Laughlin, Indiana, Indiana University Press, 2006.

DIOP Boubacar Boris, «Écrire dans l'odeur de la mort, Des auteurs africains au Rwanda », in Revue Lendemains, revue trimestrielle, Littérature africaine et génocide : de l'engagement au témoignage, 2004, pp. 73-81.

DERRIDA Jacques, « Survivre/Journal de bord » dans Parages, Paris, Galilée, 1986. 
LADMIRAL Jean-René, Traduire : théorèmes pour la traduction, Paris, Gallimard, 1994.

LADMIRAL Jean-René et MESCHONNIC Henry, « Poétique de.../Théorèmes pour la traduction : éléments d'une traduction philosophique », in Langue française, $\mathrm{n}^{\circ}$ 51, sept. 1981.

LEDERER M., La traduction aujourd'hui, Paris, Hachette, 1994.

MEYER Michel, La rhétorique, Paris, P. U. F., 2004.

\section{NOTES}

1. Traduction : «J'ai moi-même souvent vu à la télé des scènes difficiles à supporter. Des types en combinaisons et masques, en train d'extraire les corps d'un charnier. Des nouveau-nés qu'on balance en rigolant dans des fours à pain. Des jeunes femmes qui enduisent leur cou d'huile avant d'aller au lit. Elles disent : comme ça, quand les égorgeurs viendront, la lame de leur couteau fera moins mal » (Diop, $2001:$ 17).

2. Traduction : «Eh bien, c'était à Gitarama, où nous étions les plus forts, nous les Hutus. Pendant que les nôtres étaient occupés à piller et à violer, un enfant de quatre ans et ses parents attendaient une voiture pour s'enfuir en direction de Mutara. Soudain, nos hommes ont vu cette famille d'Inyenzi monter précipitamment dans la voiture. Ils ont couru, couru. C'était trop tard. Voilà comment ces imbéciles ont laissé échapper, il y a trente-sept ans, le gamin qui est aujourd'hui le chef de la guérilla »(Diop, $2001: 23$ ).

3. Traduction : « Depuis 1959, chaque jeune Rwandais doit, à un moment ou à un autre de sa vie, répondre à la même question: faut-il attendre les tueurs les bras croisés ou tenter de faire quelque chose pour que le pays redevienne normal ?» (Diop, $2001: 41$ ).

4. Traduction: «C'est vrai : si nous n'arrivons pas à éliminer tous les Tutsi, nous serons les méchants de l'histoire. Ils serviront au monde entier des lamentations bien orchestrées et ce sera drôlement compliqué pour nous. Même les moins résolus d'entre nous le savent: après le premier coup de machette, il faudra absolument aller jusqu'au bout » (Diop, $2001: 22$ ).

\section{RÉSUMÉS}

La traduction en anglais de Murambi, le livre des ossements de B.B. Diop par Fiona Mc Laughlin, Murambi : the Book of Bones, pose la question du changement de langue qui permet de s'adresser à un nouveau lecteur. Le présent article se penche sur la problématique de la traduction de l'horreur, de la mémoire et de la narrativité du génocide rwandais. Nous montrerons que dans la version anglaise, la traduction garde implicitement la même teneur du texte source mais introduit des éléments qui relèvent de la problématologie du changement d'auteur, de narrateur et de lecteur. Nous verrons que par le biais de la narratologie, le traducteur altère l'ethos du narrateur et des stratégies discursives et de figurativité, par le logos, afin de susciter le pathos.

The English translation (Murambi: the Book of Bones by Fiona Mc Laughlin) of Murambi, le livre des ossements raises the issue of language change which allows the translation to target a new audience. This article focuses on the question of translation as a problematology of horror, memory and narrative of the Rwandan genocide. The English version implicitly maintains the 
same content of the source text while roughly raising the problematology of author, narrators, and audiences changes. We show that, through the narratological problem, the translator alters more or less the ethos of the narrator and discursive strategies and figurativity by the logos in order to generate the pathos.

INDEX

Mots-clés : traduction, Murambi-le livre des ossements, Murambi - the Book of Bones, B.B. Diop, Fiona Mc Laughlin, ethos, pathos, logos

Keywords : translation, Murambi - the Book of Bones, B.B. Diop, Fiona Mc Laughlin, ethos, pathos, logos

\section{AUTEUR}

\section{BABOUCAR DIOUF}

Université Gaston Berger - Saint-Louis - Sénégal 